\title{
Radio Over Ethernet (RoE), Architecture And Design Considerations Hospital
}

\section{Mohammad Javad Sayadi*1무, Juan Sebastián Rodríguez Páez²}

${ }^{1}$ PhD Student in Medical Informatics, Department of Health Information Management, School of Health Management and Information Sciences, Iran University of Medical Sciences, Tehran, Iran.

2 Interconnect Design Engineer at Mellanox Technologies, Copenhagen, Capital Region, Denmark

\begin{tabular}{ll}
\hline Article Info & A B S T R A C T \\
\hline $\begin{array}{l}\text { Article type: } \\
\text { Research }\end{array}$ & $\begin{array}{l}\text { Introduction: } \\
\text { The evolving mobile networks are envisioned to have a flexible and reliable } \\
\text { netwicle History: }\end{array}$ \\
$\begin{array}{l}\text { Received: 2019-01-03 } \\
\text { Revised: 2019-01-27 }\end{array}$ & network to meet all design requirements needed for the fifth generation \\
Accepted: 2019-02-04 & BBUs to the central office. This comes with new challenges that are \\
& addressed by new technologies like Radio over Fiber and now Radio over \\
& Ethernet, which proposes a technique to transmit all radio data types over a \\
* Corresponding author: & traditional Ethernet based front-haul network. In this paper, we focus on the \\
Mohammad Javad Sayadi & architecture and the design considerations of Radio-over-Ethernet to have a \\
Information Management, School of & more flexible and reliable front-haul network in Centralized Radio Access \\
Health Management and & Network.
\end{tabular}
Information Sciences, Iran University of Medical Sciences, Tehran, Iran.. E-mail:mjsayadi@tvu.ac.ir

\section{Material and Methods:}

In this paper we tried to use Ethernet protocol as a universal and public network protocol in radio based networks to make it flexible and reliable. This combination allows us to focus only on the access points and propose a new architecture to encapsulation (retrieve) radio data into (from) an Ethernet frame.

\section{Results:}

This study resulted in a new architecture for radio access networks to disseminate radio data over a reliable network protocol and infrastructure. Some Ethernet header fields was modified and a mapper was included into the model in BBUs to create an adaption between radio and Ethernet infrastructures.

\section{Conclusion:}

The result shows that although this new architecture may apply additional overhead in both information and process, but having an independent fronthaul network is a necessity for Centralized Radio Access Network. However if industry implements this architecture and its processes regarding latency requirement, Radio over Ethernet will be a revolution in Centralized Radio Access Network to meet two main key design principles in 5G.

Keywords:

Radio Over Ethernet; 5 G Mobile Networks; Cloud Radio Access Networks; The Mapper

\section{How to cite this paper}

Sayadi MJ, Rodríguez Páez JS. Radio Over Ethernet (RoE), Architecture And Design Considerations Hospital. Front Health Inform. 2019; 8(1): e6. DOI: 10.30699/fhi.v8i1.170

\section{INTRODUCTION}

As we move forward to the 5th Generation (5G) of mobile networks, a revolutionary evolution has taken place in both back- and front-haul networks technologies. 5G networks [1] are expected to enable a complete mobile and connected society, by achieving a great growth in connectivity and density of traffic beyond the traditional networks [2]]. Several wireless platforms and technologies have been proposed and developed to have a soft migration to $5 \mathrm{G}$ networks.

Firstly, the general access network architecture has started to evolve form a distributed one (D-RAN) to a more centralized or cloud radio access network (CRAN). These implementations are aiming to take out 
the more complex processes from the radio access unit (RAU) and move them to the central office. This change on the network targets to reduce the latency and reduce the operation (OPEX) and implementation (CAPEX) costs of the network.

Secondly, Radio-over-Fiber (RoF) has appeared to support the distribution of the signals in these scenarios. In RoF, the wireless signals are directly modulated in the optical domain and transported to the different RAUs, where they are converted to the electric domain and transmitted. This allows using the same distribution of fiber and the high capacity of fiber communications to feed the access units, both increasing the transmitted data rate and easing the migration to $5 \mathrm{G}$.

Finally, technology that has emerged to answer some of the challenges of the future networks is Radioover-Ethernet (RoE). In RoE, the wireless signal is mapped into an Ethernet packet, and transmitted within the standards of Giga Ethernet (GE), allowing for a more robust transmission and coordinated transmission of the signals from the central office to the RAUs $[\underline{3}, \underline{4}]$.

In this paper, we begin in section 2 with a brief review of the requirements of $5 \mathrm{G}$ networks and then in section 3 C-RAN, its advantages and challenges are discussed. Afterward in section 4, Radio over Ethernet (RoE) as a novel solution toward 5G networks in C-RAN is presented and explained. Section 5 explains its design considerations and protocol before ending in section 6 with the discussion and conclusions.

\section{REQUIREMENTS OF 5G NETWORKS}

Two main key design principles guide all 5G requirements and technical solutions [2]]. Firstly, communication in future $5 \mathrm{G}$ networks will need to be flexible enough to accommodate all user applications and future extensions without increasing the complexity of management. Secondly, network should be reliable in $5 \mathrm{G}$ to deliver the required data in a required time without depending on the technology used. According to these principles, the requirements of $5 \mathrm{G}$ are classified as the following [ $\underline{5}]$ :

- High system capacity: The 5G technology should allow high reliability rates of $99.999 \%$, or higher for the use cases that demand it.

- Reduced latency: 1 millisecond is the maximum amount of latency which is acceptable in 5G.

- High data rate: When we assume tens of thousands users in crowded area, several tens of Mbps should be supported in 5G.

- Connectivity for massive devices: $5 \mathrm{G}$ should support several hundred thousand simultaneous connections per cube kilometer.

- Energy saving and cost reduction: It is important to the economic and ecological sustainability of the industry to minimize power consumption in network and terminal devices.

These requirements should be pursued by operators today using a range of techniques and are based on the operator vision of $5 \mathrm{G}$ in 2020 as well as beyond 2020.

\section{MIGRATION TOWARD 5G NETWORKS}

C-RAN is one of the recent evolutions in cellular networks toward 5G which tries to address all the challenges the operators face while supporting the increasing number of users in their networks []]. Fig 1 presents the evolution between the D-RAN and the C-RAN implementation. In C-RAN, baseband processing is centralized and shared among sites in a virtualized BBU pool, so fewer BBUs are needed in CRAN compared to the traditional architecture [7]. Due to this decrease in the number of BBUs, power and energy consumption are reduced and results in decreasing the cost of network operation []].

(a)

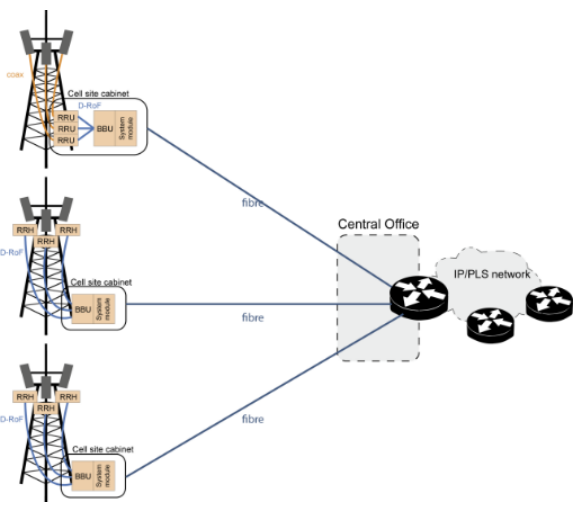

(b)

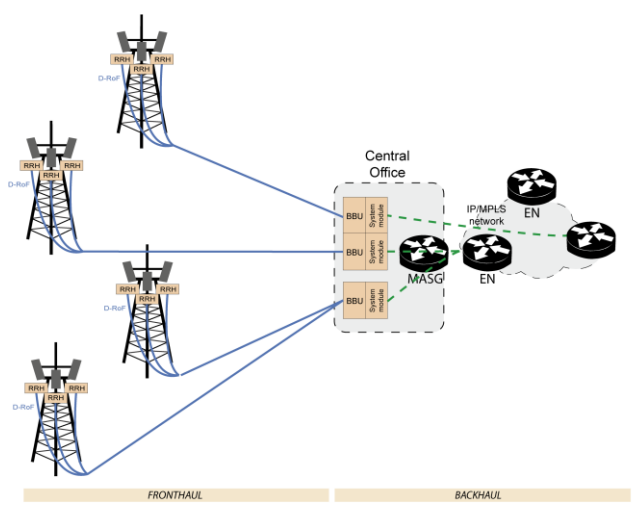

Fig 1: D-RAN (a) and C-RAN (b) architectures 
C-RAN is scalable and can be adapted to several forms of traffic, so upgrading and maintenance is much easier in comparison to the traditional RAN. More over thanks to the centralized baseband processing units and new technologies such as RoF, C-RAN is able to increase network throughput []].

Beside these advantages, C-RAN suffers from some challenges that must be considered. High bandwidth and strict latency and jitter are its basic necessities that should be achieved by a low cost infrastructure [8]. Another important problem refers to BBUs cooperation and interconnection, because C-RAN does not have a green and soft solution to coordinate all network types (WLAN, TD-LTE, GSM, GPRS, EDGE and TD-SDMA) [11].

Some researches and implementations have been done to address these challenges. $\operatorname{RoF}[\underline{11}, \underline{12}]$ is one of these technologies. Fig 2 shows exemplifies the concept of RoF. It involves modulating the radio frequency (RF) subcarrier onto an optical carrier for distribution over a fiber network link. Enormous bandwidth (up to $\mathrm{THz}$ ), high speed signal processing and low loss transmission are main advantages of using fiber. The main goal in RoF is to meet the bandwidth and latency requirements [13].

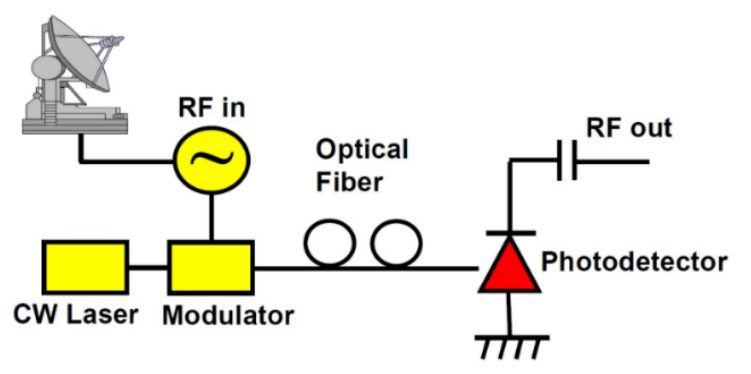

Fig 2: RoF overview

Recently, Radio over Ethernet (RoE) has been proposed as a novel idea, to achieve a cost efficient and green solution for C-RAN challenges. RoE tries to interconnect BBUs to cooperate with each other. In addition, the processing power of RRHs will be increased [14].

\section{RADIO OVER ETHERNET ARCHITECTURE}

When we want to talk about C-RAN architecture, three things are indispensable: burden on front-haul, implementation costs and flexibility of resource schedule [15].

According to the implemented architecture of C-RAN, an asymmetric function allocation between RRH and BBU has been presented. PHY layer, MAC layer and Network layer functions are implemented in BBU but RRH has only PHY layer function. Fig 3 shows the common conception of the C-RAN.

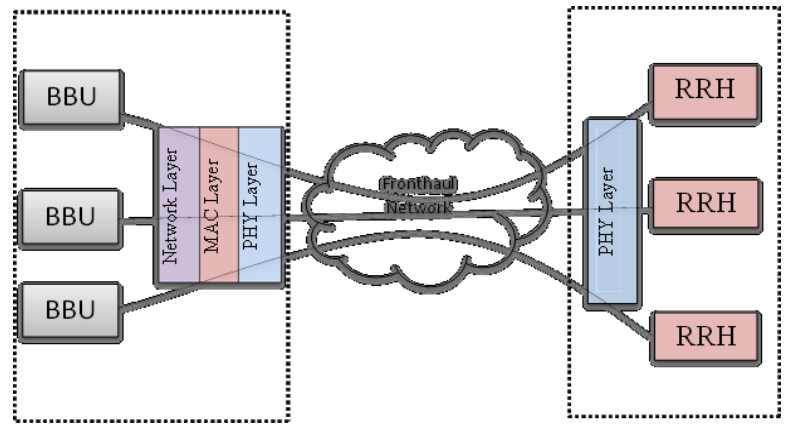

Fig 3: Current architecture of C-RAN

This architecture might have some benefits but there are some drawbacks. The burden on the front-haul and also the cost of implementation is high. On the other hand the system is unable to support coordination of different Technologies.

Radio over Ethernet, which has been standardized as P1904.3 in IEEE, tries to transfer all types of radio data across an Ethernet based network [14]. The main goal is to address the previously discussed challenges of the C-RAN:

- BBU interconnection and cooperation to coordinate all network types

- Low cost solution to have a strict latency and jitter in the network

The main scope which has been stated in P1904.3 to be followed by Radio-over-Ethernet is: All types of radio data, In-phase/Quadrature (IQ) data, possible vendor specific data and control and management channel must be digitized, packetized and encapsulated into an Ethernet frame payload field.

P1904.3 includes an adaption in physical layer to adapt data frames from radio access protocol to Ethernet data frame that is suitable for transmission over Ethernet physical media. Fig 4 shows a use case diagram for implementation of RoE.

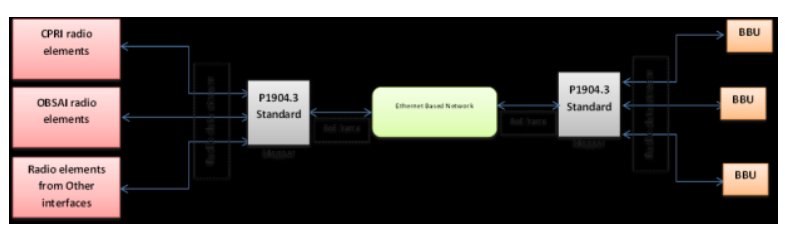

Fig 4: P1904.3 Use Case Diagram

In this use case two grey boxes named Mapper are shown for P1904.3 standard. Mapper is responsible for converting all types of data to a standard type and conversely. In this architecture each RRH should have a Mapper connected to an Ethernet switch. Another Mapper which is the same as the Mapper near to RRH should be located in BBU pool. In this architecture the Mapper is an extension to RRH to have both PHY and MAC layers. 
The first advantage of this architecture is to have an independent and flexible front-haul network, as shown in Fig 5. All radio data types are encapsulated into similar frames. So all data types from all radio access technologies have the same format and we can easily share one link to connect several radio access interfaces such as CPRI, OBSAI and etc. to BBU pool.

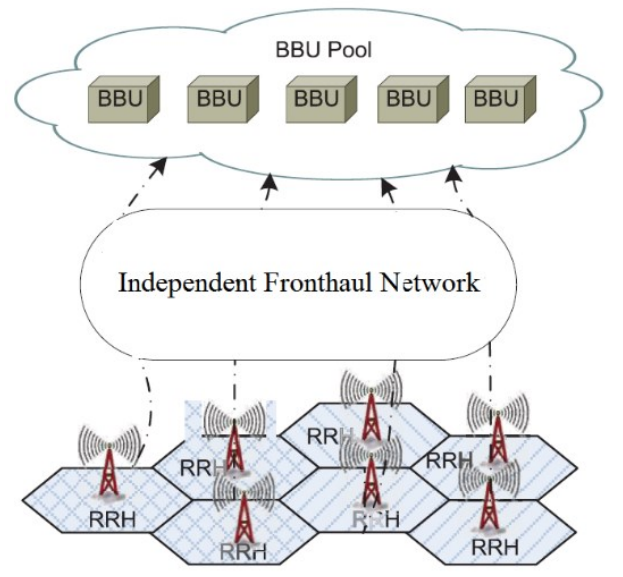

Fig 5: Frounthaul Network in C-RAN with RoE

\section{DESIGN REQUIREMENTS CONSIDERATIONS

The main question in this research is how to implement RoE architecture over current Ethernet based network. In this section firstly we will introduce Mapper as the main part of RoE and then RoE protocol will be discussed.

\section{Mapper and its design considerations}

The Mapper is the main part of RoE including suitable logic, circuitry configured to convert radio signal streams to packetized signal and vice-versa.

Having a look on Fig 3, the Mapper is located at the edge of front-haul network and it can be implemented in L2 switches to support heterogeneous service with real time characteristics. As an embedded system on Ethernet switches, the Mapper should be able to cope the radio access technologies with an independent Ethernet fronthaul network. In detail, it should recognize different types of radio data and packetize them into similar shape frames and then feed an Ethernet switch with Ethernet frames including packetized radio data as payload. However this system leads to some additional framing overhead that will be explained in section 3.2.

On the other hand Ethernet switches should recognize RoE frames so one free value should be defined for Ether_Type in Ethernet header to differentiate RoE frames from other Ethernet frames.

In addition, the Mapper in BBU side should be able to manage communication between BBUs. In this way the Mapper stablish a connection between BBUs without passing the data thorough Ethernet based network and works as a bridge between BBUs whenever they want to communicate with each other.

\section{RoE Protocol}

The main part of RoE is the encapsulation of radio data into an Ethernet frame. To have an efficient protocol to encapsulate the radio data, End to End Delay, as the most important issue, should be considered.

The general scenario in the source Mapper includes the following steps:

Obtaining the radio data and creating a 20-Byte Basic Frame including 16 Bytes for data and 4 Bytes for header, shown in Fig 6.

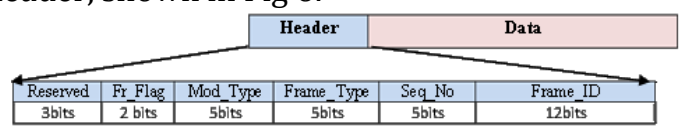

Fig 6: RoE basic frame

Frame_ID is a 12 bits field to identify radio frames. Starting with 0 and when it reaches 4095, then it will be set by 0 . Each radio frame gets one Frame_ID. If we have fragmentation for frames larger than 16 Bytes, all fragments have similar Frame_ID and they will be differentiated by Seq_No.

Since the largest frame in CPRI is 324 Bytes long so Seq_no should be 5 bits long. When fragmentation taken place, this field helps to recognize pieces of on radio frame. To manage the fragmentation and defragmentation process a 2-bit field named Fr_Flag has been used. The possible values of Fr_Flag and their meanings are shown in Table 1.

Table 1: Possible values of fragmentation flag

\begin{tabular}{|c|c|c|c|c|}
\hline Value & 11 & 10 & 01 & 00 \\
\hline Meaning & $\begin{array}{c}\text { Whole radio data } \\
\text { within the payload }\end{array}$ & $\begin{array}{c}\text { Start of ractio } \\
\text { data }\end{array}$ & $\begin{array}{c}\text { End of radio } \\
\text { data }\end{array}$ & $\begin{array}{c}\text { Middle part of } \\
\text { radio data }\end{array}$ \\
\hline
\end{tabular}

Frame_Type shows the type of frame. The system is able to support several types of radio data regardless their technology and interface.

Mod_Type shows the type of interface or system that has produced the radio signal.

1. Creating an Ethernet frame with the following features:

2. Ether_Type $=$ 'E001'. This value is free now and could be used for RoE frame. 1500-Byte payloadfield (Fig 7). 


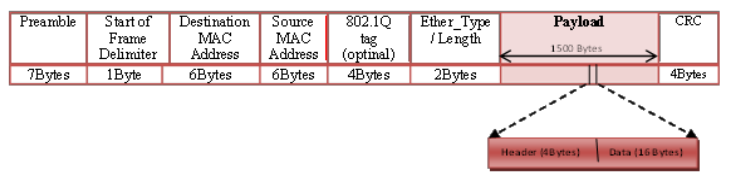

Fig 7: RoE general packet

We can use Type='00' in Basic Frame for invalid frames. This can helps when we have less than 75 frames in the buffer.

Send the created Ethernet frame into an Ethernet based network.

The general scenario in the destination includes the following steps:

1. Extracting the received Ethernet frame and obtaining the payload.

2. Breaking down the payload into 75 similar segments which are basic frames.

3. Obtaining data and header section for each basic frame.

4. If Fr_Flag=11 that mean there is no fragmentation so the radio frame can be sent.

5. If fragmentation is needed, the data should be put into a buffer to manage the defragmentation process.

The mapper can be a middleware including above functionalities and implemented as a part of Ethernet switches.

\section{Delay analysis}

The key challenge in C-RAN technology that must be considered in all new protocols and evolutions is delay. As an important necessity in C-RAN we should have an analysis to show our protocol meets this restriction.

According to [8] the round trip delay requirement of the user plane data (IQ Data), not including the medium propagation delay, is $5 \mu \mathrm{s}$. In terms of calibration the accuracy should satisfy \pm 16.276 ns [17].

To measure the delay we should focus on transmission and packetizing delay in mapper and also latency increased by Ethernet switches. To satisfy this requirement in C-RAN these delays must be in Nano second scale.

According to [16] if we use a switch with $100 \mathrm{Gbps}$ interface, the transmission delay is $0.01 \mathrm{~ns}$. Since we have 27 percent header for the original radio data, the maximum amount of transmission delay is $0.05 \mathrm{~ns}$ per each bit and 6.4 ns per each user data sub frame. The switch processing delay for a 1518-Byte frame in maximum is less than 20ns. Regarding our protocol,
75 user data sub frames or 9600 bits in each Ethernet frame, the processing delay is $0.5 \mathrm{~ns}$ per user data sub frame and 4ps per bit.

The packetizing delay in mapper is the effective time in round trip delay measurement. This delay can be minimized under $10 \mathrm{~ns}$ if thoroughly is implemented in hardware level. Because the radio data is opaque to the mapper and the system does not read its content, implementing the mapper to packetize the radio data in hardware level is not too complicated. All in all, the summation of transmission, mapper packetizing and switch processing delays should not exceed the amount of accuracy.

\section{CONCLUSION}

We have presented architecture and its design considerations for Radio-over-Ethernet to have a flexible and reliable front-haul network in C-RAN. The mapper as the main part of RoE encapsulate all radio data types into a unique-form Ethernet frame and transmit it over an Ethernet based front-haul network and make the front-haul network independent from the technologies used in back-haul or in RRH.

Although this new architecture may apply additional overhead in both information and process, but having an independent front-haul network is a necessity for C-RAN. However if industry implements this architecture and its processes regarding latency requirement, RoE will be a revolution in C-RAN to meet two main key design principles in 5G.

\section{AUTHOR'S CONTRIBUTION}

All the authors approved the final version of the manuscript.

\section{CONFLICTS OF INTEREST}

The authors declare no conflicts of interest regarding the publication of this study.

\section{FINANCIAL DISCLOSURE}

No financial interests related to the material of this manuscript have been declared.

\section{REFERENCES}

1. Checko A, Christiansen H, Yan Y, Scolari L, Kardaras G, Berger $\mathrm{M}$, et al. Cloud RAN for mobile networks: A technology overview. IEEE Communication Surveys \& Tutorials. 2015; 17(1): 405-26.

2. Hattachi RE, Erfanian J. 5G white paper: Next generation mobile networks. NGMN Alliance; 2015.

3. Umesh A. Study on new radio access technology: Radio access architecture and interfaces. Report No: TR 38.801, v14.0.0, Release 14. 3GPP; 2017.

4. Sayadi M, Rodrigez J, Olmos J, Monroy I. Latency and 
bit-error-rate evaluation for radio-over-ethernet in optical fiber front-haul networks. Journal of Optical Switching and Networking. 2018; 27: 88-92.

5. Cicconetti C. 5G radio network architecture: Radio access and spectrum [Internet] 2017. [cited: 1 Jul 2018]. Available from: http://www.ict-ras.eu.

6. Jose S. Visual networking index. Global mobile data traffic forecast update, 2012-2017. USA. 2013.

7. Wang R, Hu H, Yang X. Potentials and challenges of CRAN supporting multi-RATs toward 5G mobile networks. IEEE Access. 2014; 2: 1187-95.

8. C-RAN the road towards green ran. China Mobile Research Institute; 2011.

9. Brown G. Converging telecom \& IT in the LTE RAN. Samsung; 2013.

10. Irmer R, Droste $H$, Marsch P, Fettweis G, Brueck S, Mayer $\mathrm{H}$, et al. Coordinated multipoint: Concepts, performance and field trial results. IEEE Communications Magazine. 2011; 49(2): 102-11.

11. Lee SL, Chuang $\mathrm{MH}$, Lee $\mathrm{CH}$, Sun $\mathrm{CH}$, Tseng, MingChien. Design and applications of radio-over-fiber schemes to indoor wireless coverage and high-speed rails. 3rd International Conference on Photonics. 2012; IEEE.

12. Lim C, Nirmalathas A, Bakaul M, Gamage P, Lee L, Yang $Y$, et al. Fiber-wireless networks and subsystem technologies. Journal of Lightwave Technology. 2010; 28(4): 390-405.

13. Cavalcante L, Silveira LFQ, Rommel S, Olmos J, Monroy I. Performance analysis of wavelet channel coding in COST207-based channel models on simulated radioover-fiber systems at the w-band. Opt Quant Electron. 2016; 48:28-37.

14. IEEE standard for radio over ethernet encapsulations and mappings. IEEE; 2015.

15. Sabella D, Rost $P$, Sheng $Y$, Pateromichelakis E, Salim U, Guitton-Ouhamou P, et al. RAN as a service: Challenges of designing a flexible RAN architecture in a cloud-based networks. Conference on Future Networks \& Mobile Summit. IEEE; 2013.

16. Cisco Nexus 9516 scaling $100 \mathrm{G}$ performance to new heights [Internet]. 2015. [cited: 1 Jul 2018]. Available from: http://networktest.com.

17. Common Public Radio Interface (CPRI): Interface Specification. CPRI Publications; 2013. 